\title{
Thomas der Lügner - und seine Geistesmenschen. Vom Lebenswerk als Lebenslüge
}

Thomas le menteur - et ses « Geistesmenschen ». De l'ouvre comme mensonge vital

Thomas the Liar - and his "Spiritual Men" ("Geistesmenschen »). A Life's Work as a Lifelong Lie

\section{Paola Bozzi}

\section{OpenEdition}

\section{Journals}

Édition électronique

URL : http://journals.openedition.org/ceg/1569

DOI : $10.4000 /$ ceg. 1569

ISSN : 2605-8359

\section{Éditeur}

Presses Universitaires de Provence

Édition imprimée

Date de publication : 15 juin 2015

Pagination : 207-217

ISBN : 978-2-85399-993-9

ISSN : 0751-4239

\section{Référence électronique}

Paola Bozzi, «Thomas der Lügner - und seine Geistesmenschen. Vom Lebenswerk als Lebenslüge », Cahiers d'Études Germaniques [Online], 68 | 2015, Online erschienen am: 17 Dezember 2017, abgerufen am 02 Dezember 2020. URL : http://journals.openedition.org/ceg/1569 ; DOI : https://doi.org/ 10.4000/ceg.1569 


\section{Thomas der Lügner - und seine Geistesmenschen. Vom Lebenswerk als Lebenslüge}

Paola BOZZI

Università degli Studi di Milano

Die Lebenslüge, die ist das stimulierende Prinzip

H. J. Ibsen

In seinen autobiografischen Jugenderinnerungen erzählt Thomas Bernhard gnadenlos von der Schande seiner unehelichen Geburt und vom Verstoß durch die Mutter, die ihn als infamen Lügner bezeichnete. ${ }^{1}$ Er fügt hinzu, er ,,habe zeitlebens immer die Wahrheit sagen wollen“, auch wenn er inzwischen wisse, „es war gelogen“ (KE 33). Denn der Wille zur Wahrheit führe, so Bernhard, unausweichlich zu ihrer Verfälschung (KE 42f.). Gerade dieses Spannungsfeld zwischen Wahrheit und Lüge prägt das ganze Euvre des Autors. In seinem Mittelpunkt steht ein bestimmter Typus, eine literarische Figur, deren Selbstverständnis und Identität weitgehend auf den ,Geist' reduziert ist. Der Geistesmensch ist Vertreter der Theorie, dass menschliche Größe und Werthaftigkeit nur dem Einzelnen zukomme in Opposition zum überwiegenden Teil der Durchschnittsmenschen; er ist dabei jener „Wahrheitsfanatiker“ (K 15) bzw. „Störenfried“ (K 124), der der Welt Wahrheiten verkündet, die sie nicht hören will - eine Definition, die durchaus auf das Selbstverständnis des Autors verweist. Zu seinen Existenzialien gehört weiterhin eine gewisse Affinitität zur Krankheit. Während diese für den Massenmenschen eine Beeinträchtigung des Lebens ist, stellt sie für den Geistesmenschen eine fast schon prophetische Steigerung dar: „Der Kranke ist der Hellsichtigste, keinem anderen ist das Weltbild klarer" (AT 48). Der Geistesmensch definiert sich also durch die Abgrenzung von anderen: Sein Selbstbewusstsein ist das Bewusstsein, anders (besser) zu sein, das Grundmotiv all seiner Bestrebungen ein tief verwurzeltes Bedürfnis

Thomas Bernhard, Ein Kind, Frankfurt a. M., Suhrkamp, 1982 (K), S. 27; im Folgenden werden die Texte Bernhards direkt im Text mit der entsprechenden Abkürzung und Seitenangabe zitiert: Der Keller. Eine Entziehung, Salzburg, Residenz, 1976 (KE); Korrektur [1975], Frankfurt a. M., Suhrkamp, 1988 (KO); Das Kalkwerk [1970], Frankfurt a. M., Suhrkamp, 1971 (KA); Auslöschung. Ein Zerfall, Frankfurt a. M., Suhrkamp, 1986 (A); Beton, Frankfurt a. M., Suhrkamp, 1982 (BET); Der Atem. Eine Entscheidung, Salzburg/ Wien, Residenz, 1978 (AT). 
nach Unverwechselbarkeit und Anerkennung als Individuum. Rigoros verfolgt er deshalb den Entwurf einer radikal geistigen Existenz, die auf die Emanzipation von der als „Marter“ empfundenen Herkunft (KO 272) bzw. einer vorgegebenen Welt voller Fremdheit und Unverständnis einerseits, auf totale Erkenntnis und Wahrheit andererseits zielt. So setzt er sich nicht nur von diesem Territorium ab, sondern erschafft sich im gleichen Zuge eine eigene ,Lebens-Welt' (KO 237-239).

Ausnahmslos Männer unterstellen in Bernhards Texten ihre gesamte Existenz einer lebenslangen, fruchtlos bleibenden künstlerisch-intellektuellen Arbeit, die alle Kräfte des Individuums fordert. Sie stellen all jene Eigenschaften unter Beweis, die gemeinhin als Voraussetzungen des Gelingens angesehen werden: Ausdauer, Disziplin, Selbstlosigkeit, Mut. ${ }^{2}$ Bernhards Figuren beschäftigen sich meist privat mit Studien und Experimenten. Sie machen sich eine Unmenge von Notizen (KO 7) und ziehen sich für ihre ,poetisch-wissenschaftliche Faszination“ (V 47) in die „Einsamkeitszelle“3 eines „Arbeitskerkers“ (KA 33) bzw. einer „Denkkammer“ (KO 23) zurück. Die solipsistische Abkehr von der sinnlichen und sozialen Wirklichkeit, die Weltlosigkeit einer verkopften Existenz wird oft bei Bernhard als freiwillige Einkerkerung in eine symbolische Örtlichkeit, einen locus intellectualis verdeutlicht. Exemplarisch petrifiziert das Kalkwerk im gleichnamigen Roman jene Gegebenheiten, die der Geistesmensch Konrad unabdingbar für seine Arbeit zu brauchen vorgibt und deshalb durch zusätzliche bauliche Maßnahmen und künstliche Überformung noch verstärkt. Wo die Gesellschaft gleichbedeutend ist mit Materialismus, Dummheit und sinnlichem Exzess, soll eine solche Enklave die Herrschaft des Geistes, Askese, (Selbst)Zucht und Disziplin bezeichnen. Denn Bernhards Geistesmenschen lehnen es ab, das ihnen leicht Erreichbare überhaupt realisieren $\mathrm{zu}$ wollen und ringen um Großes, das den Lebenssinn setzt, um das Lebenswerk. Dabei werden die höchsten Ansprüche als Bedingungen gestellt, was den Anschein eines Geniekonzepts ${ }^{4}$ erweckt (KA 61). So visiert der Protagonist von Auslöschung lebenslang ein „Buch über alle Wahrnehmungen“, ein Buch, über Alles“ und ,über das ganze Mögliche“ (A 55) an, das dem totalen Geistesprodukt gleicht.

Die Konzentration auf Wissenschaft und Kunst soll sich meist in der Form

So untersucht Willi Huntemann Bernhard und Beckett im Hinblick auf ihre Zugehörigkeit zur Moderne resp. Postmoderne; vgl. seinen Aufsatz „Treue zum Scheitern. Bernhard, Beckett und die Postmoderne“, in Heinz Ludwig ARnold (Hrsg.), Thomas Bernhard, München, Edition Text [und] Kritik, 1982, S. 42-74.

Vgl. Jens Tismar, Gestörte Idyllen. Eine Studie zur Problematikderidyllischen Wunschvorstellungen am Beispiel von Jean Paul, Adalbert Stifter, Robert Walser und Thomas Bernhard, München, Hanser, 1973 [zugl.: Berlin, Diss., 1972], S. 106.

4 Insbesondere der Begriff des ,Genies' bildete ein wesentliches Schlagwort des Nietzsche-Kults, der ab Mitte der 80er Jahre des 19. Jahrhunderts einsetzte und den Philosophen zum Propagator von Männlichkeit machte. Den Wunsch nach einer ,männlichen' Erneuerung der Kultur hatte Friedrich NiETzSCHE bereits in der Geburt der Tragödie (1872) formuliert (vgl. Kritische Studienausgabe, hrsg. von Giorgio Colli und Mazzino Montinari, München, Dt. Taschenbuch-Verl., 1988, S. 526; vgl. dazu auch Urte Helduser, Geschlechterprogramme. Konzepte der literarischen Moderne um 1900, Köln/ Weimar/ Wien, Böhlau, 2005, S. 88-90); in den 1886 unter dem Titel Jenseits von Gut und Böse erschienenen Aphorismen beklagte NIETZSCHE weiterhin eine übersteigerte Idealisierung des Weiblichen, die „Entmännlichung der Kunst“ (Kritische Studienausgabe, S. 52). 
eines Werks bzw. einer Studie konkretisieren ${ }^{5}$, welche der Autor oft und unablässig als Thema in seinen Texten aufnimmt. Nicht nur Das Kalkwerk und Korrektur, sondern auch Frost, Amras, Verstörung, Watten und Gehen ${ }^{6}$, d.h. fast alle großen Prosawerke in der frühen Phase Bernhards, behandeln die Problematik der Studie, die er auch in den späteren Werken Ja, Die Billigesser, Beton, Der Untergeher ${ }^{7}$ und zuletzt Auslöschung fortsetzt. Denn Schreiben ist sowohl privilegierter Ort der Transgression des Selbst, als auch der Konstituierung und Selbstvergewisserung. Es erhält die Funktion einer techne, einer Gestaltungstechnik, die dem Schreibenden verspricht, zu sich selbst zu kommen oder aber das als Begrenzung empfundene sich selbst zu überwinden - kurz: Es fungiert als Selbst- oder Subjekttechnologie im Sinne Foucaults. ${ }^{8}$ Bei Bernhard geht es aber dabei immer um sehr ungefähre Projekte, über deren Thema und Aufbau der Leser nichts erfährt. In Beton unterstreichen die große Anzahl der bereits geplanten Schriften und die Verschiedenheit ihrer Themen, dass für den Geistesmenschen Rudolf die Wahl des jeweiligen Gegenstandes nur eine relative Bedeutung haben kann. Bevor er sich seinem „Lieblingskomponisten“ Mendelssohn Bartholdy widmet, will er bereits eine Vielzahl wissenschaftlicher Arbeiten verfassen: eine „Skizze Über Schönberg“ (BET 13), eine Arbeit zu „Jenufa“, eine zu „Moses und Aaron“, eine „Schrift Über Rubinstein“, eine „Arbeit über Die Six“ (BET 14), eine Schrift über „Michael Haydn“ (BET 18), eine über „Reger“ (BET 48), eine andere über „Bach“ (BET 70) und sogar eine über „Nietzsche“ (BET 49). Diese Studien fungieren (fast) ausschließlich als Chiffren des Topos ,Lebenswerk' und verweisen auf eine Obsession des männlichen Subjekts, die keine Aussicht auf Erfüllung hat: Sie gerinnen nicht in Sprache und bleiben im Kopf (KA 71) ${ }^{9}$, befinden sich also im Stadium des rein Gedanklichen, Uneindeutigen und Unendlichen. Schon der frühe Roman Frost aus dem Jahr 1963 widmet sich der Beobachtung eines Malers, der das Malen eingestellt hat, wobei der Text gerade den Akt des Nicht-Malens als einen künstlerischen Akt interpretiert. Die Fixierung des Malers Strauch auf die Schatten in seinem Kopf kündigt an, was die späteren Romanhelden Bernhards, allesamt Geistesmenschen, kennzeichnen wird. So stellt das nie verwirklichte Werk bzw. die scheiternde, nie begonnene, nicht zu Ende gebrachte ${ }^{10}$ oder nach Vollendung

5 In den 22 größeren zwischen 1963 und 1986 entstandenen Prosatexten Thomas Bernhards zählt Christoph BARTMANN mehr als 11 „,Studien’, an denen Figuren innerhalb fiktionaler Zusammenhänge arbeiten“. Von diesen kommt der allergrößte Teil nicht zustande; vgl. seinen Aufsatz „Vom Scheitern der Studien. Das Schriftmotiv in Bernhards Romanen“, in Heinz Ludwig Arnold, Thomas Bernhard, S. 2228, hier S. 22.

6 Thomas Bernhard, Frost, Frankfurt a. M., Insel-Verl., 1963; Amras, Frankfurt a. M., Insel-Verl., 1964; Verstörung, Frankfurt a. M., Insel-Verl., 1967; Watten. Ein Nachlaß, Frankfurt a. M., Suhrkamp, 1969; Gehen, Frankfurt a. M., Suhrkamp, 1971.

7 Thomas Bernhard, Ja, Frankfurt a. M., Suhrkamp, 1979; Die Billigesser, Frankfurt a. M., Suhrkamp, 1980; Der Untergeher, Frankfurt a. M., Suhrkamp, 1983.

8 Vgl. Sylvia Pritsch, Rhetorik des Subjektes. Zur textuellen Konstruktion des Subjekts in feministischen und anderen postmodernen Diskursen, Bielefeld, Transcript-Verl., 2008, S. 37.

9 Alexandra Pontzen sieht in dieser Situation keine Lebenslüge, sondern eine Analogie zu einer Schwangerschaft; vgl. ihre Monographie Künstler ohne Werk: Modelle negativer Produktionsästhetik in der Künstlerliteratur von Wackenroder bis Heiner Müller, Berlin, Schmidt, 2000 [Zugl.: Bonn, Univ., Diss., 1999], S. 333.

${ }^{10}$ Man denke an die zum Lebensprojekt schlechthin erhobenen Künstlerbiographien in Beton, 
durch „Korrektur der Korrektur der Korrektur der Korrektur“ (KO 361) zerstörte, verschwundene Studie (KO 178-180) nicht nur ,das Motiv der Motive Bernhards“"11, sondern durch ihre Abwesenheit das fehlende Zentrum seiner dezentrierten Texte dar und wird als Leerstelle zur suggestiv bildlosen Metapher einer Lebenslüge. Es geht bei Bernhard also nicht um ein Beispiel von ,pedantischer Pseudowissenschaft“, wie Claudio Magris behauptet ${ }^{12}$, sondern vielmehr um ein Konstrukt, das das Dasein erträglicher machen soll, um eine Täuschung, aus der der Geistesmensch den Mut schöpft, weiterzuleben. Gerade als solche stellt die Studie für den Geistesmenschen den einzigen „Existenzzweck“ (KA 16) und das wahre Kunststück dar. Darauf, dass die Studie bei Bernhard ,ausdrücklich mit dem Versuch der Selbstverwirklichung ${ }^{\text {“13 }}$ in Zusammenhang gebracht wird, hat Manfred Mittermayer bereits aufmerksam gemacht. Auch Hartmut Reinhardt deutet das unentwegte Bemühen um die Studie als einen „Versuch des Subjekts [...], sich durch die Niederschrift ,in einem einzigen Zuge' der eigenen Identität zu versichern" ${ }^{\text {"14. }}$. Wo aber das Dasein von Wahrheitsfanatismus einerseits, von Lebenslüge andererseits beherrscht ist, stellt es sich als heikle Gratwanderung zwischen luzider Einsicht und tiefem Abgrund dar.

Als Henrik J. Ibsen das Drama Vildanden im Jahr 1884 verfasste, brauchte er die Lebenslüge (livsløgn) nicht zu erfinden. ${ }^{15}$ Denn ,,[d]ie Lebenslüge ist ein Ausweg aus der im 19. Jahrhundert entstandenen Krise des Individuums "16, als der Glaube an einen Wert, der jedem Menschen per se zukommt, verloren ging. Damit ist gemeint, dass der Einzelne vor die Aufgabe gestellt wird, seinem Dasein einen allgemeinen anerkannten Wert und übergeordneten Sinn zu verleihen. Dies ist eine individuelle Aufgabe, d.h. jeder muss sie für sich selbst lösen, wobei jede erdenkliche Form von Anerkennung in Betracht zu ziehen ist. Die Anfänge dieser Krise reichen zurück ins späte 18. Jahrhundert, also in die Zeit der Aufklärung. Damals widmeten sich Denker und Autoren der Frage, ob das Selbst seinen Wert dadurch erhält, dass es sich von anderen unterscheidet oder dadurch, dass es mit der Ganzheit der Menschheit oder der Gesellschaft verschmilzt. So kommt zu den beiden Kategorien des Seins und des Habens im 19. Jahrhundert eine dritte hinzu bzw. sie gewinnt - sofern es sie vorher schon gab - an Bedeutung: die Kategorie des Machens. Das Machen im Sinne von Erschaffen ist eine ureigene Kategorie des menschlichen Befindens, das sich von den beiden anderen Grundkategorien fundamental unterscheidet. Denn es verweist

Wittgensteins Neffe (Eine Freundschaft, Frankfurt a. M., 1982) und Auslöschung, der Studien naturwissenschaftlich-philosophischer Art in Ja, Die Billigesser, Das Kalkwerk und Verstörung, des literarisch-autobiographischen Lebenswerks in Auslöschung.

11 Bartmann, ,Vom Scheitern der Studien“, S. 24.

12 Claudio Magris, „Geometrie und Finsternis“, in Études Germaniques, 33, 1978, S. 282-297, hier S. 292.

13 Manfred MittermaYer, Thomas Bernhard, Stuttgart, Metzler, 1995, S. 63.

${ }^{14}$ Hartmut ReINHARDT, „Das kranke Subjekt. Überlegungen zur monologischen Reduktion bei Thomas Bernhard“, in Germanisch-Romanische-Monatsschrift, Neue Folge, Bd. 26, 1976, H. 3-4, S. 334-356, hier S. 347.

15 Doch hat er diesem Phänomen nicht nur einen Namen gegeben, sondern er hat es überhaupt erst sichtbar werden lassen.

${ }^{16}$ Claude FeIder, Beiträge zu einer Theorie der Lüge, Freiburg, HochschulVerlag, 1999, S. 7. In diesem Sinn ist die Untersuchung an Feiders Darstellung der Problematik angelehnt. 
direkt auf die Person desjenigen, der etwas macht. Vor allem das Machen auf dem Gebiet der Kunst ist ein kreativer Prozess, der zur Entstehung eines einzigartigen, eines originellen Werks führt, womit jedoch nichts über die Qualität des Kunstwerks gesagt ist.

Im letzten Viertel des 19. Jahrhunderts wird die neue Werteskala, an der die Bildungsbürger sich weitgehend orientieren, von Nietzsche so apodiktisch festgelegt, dass ein Abweichen davon fast dem Eingeständnis eines Versagens gleichkommt. So kristallisieren sich schließlich zwei entgegengesetzte Einstellungen zum Eigenwert des Individuums heraus: Einerseits das uneingeschränkte Recht des Einzelnen, sich auf Kosten der Masse zu profilieren, sofern er die innere Kraft und das nötige Durchsetzungsvermögen hat; andererseits eine vom christlichen Gedanken der Gleichheit vor Gott getragene Demut und Selbstbeschränkung - das ist die Alternative, die sich rund hundert Jahre nach der Französischen Revolution aus den an sich konträren Forderungen nach Gleichheit und Freiheit heraus ergibt. Vor allem innerhalb des Bildungsbürgertums gibt man sich nicht länger mit dem Heldenkult und der Verehrung , großer Männer' zufrieden, sondern immer mehr Bildungsbürger haben offen oder insgeheim den Wunsch und das Bedürfnis, ebenfalls zur Elite zu gehören und ihrem Leben einen hohen Wert zu verleihen. Damit steigt aber auch die Zahl der Mitstreiter, und die Schwierigkeit, sich von anderen im positiven Sinne abzuheben, nimmt im gleichen Maße zu wie die Steigerung des Durchschnittswertes. Ein Leben, so scheint es, hat nur einen Sinn, wenn es der betreffenden Person gelingt, etwas zu tun, was sie von anderen unterscheidet. Die anderen bilden die Folie, von der sich der Einzelne abheben will. Sie sind die notwendige Matrix, das Dunkle, dessen Kontrast das Helle überhaupt erst sichtbar werden lässt.

Wenn aber bereits im Hinblick auf das ausgehende 19. Jahrhundert von einer Hypertrophie des Geltungsbedürfnisses innerhalb des Bildungsbürgertums die Rede ist, so kann diese Bezeichnung zur Charakterisierung der Verhältnisse in den westlichen Ländern nach 1968 auf weite Teile der Bevölkerung ausgedehnt werden. Denn im gleichen Maße wie das allgemeine Bildungsniveau gestiegen ist, hat auch der Wunsch nach Anerkennung als Individuum zugenommen. Dabei spielt dieses Streben nach Sich-Vergleichen und Sich-Unterscheiden-Wollen eine viel größere Rolle in der Motivation des Machens auf geistigem Gebiet als es generell den Anschein hat und als viele es wahrhaben bzw. zugeben wollen. Obwohl die geistigen Anlagen vorhanden sein müssen, ist der Anspruch des Individuums auf Anerkennung nicht auf das Sein gegründet, auch nicht auf das Haben, sondern allein auf das Machen, auf die von ihm erbrachte intellektuelle Leistung. Dem Durchschnittsbildungsbürger bleiben so nur zwei Auswege: entweder schraubt er seine Ambitionen zurück und findet sich damit ab, ein Angehöriger des Mittelmaßes zu sein, so dass ihn, wenn er die Überlegenheit anderer nicht anerkennen will, nichts anderes übrig bleibt, als an die Gleichheit qua Gleichwertigkeit aller Menschen zu glauben, oder aber er baut sich eine Lebenslüge auf und das heißt, dass er - ohne erkennbaren Grund - von seiner eigenen Überlegenheit und Einzigartigkeit überzeugt ist. Im Unterschied zu einem Menschen, dessen Alltagsleben von seinem Wunschtraum streng getrennt ist, baut der Lebenslügner sein gegenwärtiges Leben auf einer falschen oder zumindest unrealistischen Vorstellung von der eigentlichen Bestimmung seines Lebens auf. Er 
gestaltet sein Leben im Hinblick auf ein höheres Ziel, das er jedoch nie erreichen wird, weil er auch gar nicht erst den Versuch unternimmt, es zu erreichen. In diesem Sinne richtet der Geistesmensch bei Bernhard seine Lebensführung so aus, dass sie mit seiner Vorstellung von einer großen Lebensaufgabe nicht kollidiert. Die jeweils spezielle Zufluchtsstätte des einsam arbeitenden Geistesmenschen, das äußerlich zur Festung aufgerüstete und innen klösterlich karge Kalkwerk verkörpert zwar exemplarisch, wie explizit beabsichtigt, die Bedingung der Möglichkeit der Studie, erweist sich aber zugleich als Garant der Unmöglichkeit ihrer Niederschrift. Denn die äußere Ruhe führt bald zur inneren Unruhe, schlägt also um und erzielt die gegenteilige Wirkung (KA 94-95): Die Enklave entpuppt sich als Ort der Ohnmacht.

Damit rückt im Text nicht das Werk bzw. die Schrift selbst, sondern die Reflexion über das Scheitern des Protagonisten an der Realisierung seiner künstlerischwissenschaftlichen Projekte ins thematische Zentrum. Der Geistesmensch ist darum bemüht, die Ursachen für das Scheitern auszumachen, kommt aber letztlich zu keinem zufriedenstellenden Ergebnis. Alle angeführten Erklärungen und Entschuldigungen für die Verhinderung der Niederschrift enthalten den Grundton des Zweifelhaften: kaum, dass die wirkliche Ursache gefunden zu sein scheint, wird sie im Zuge eines Rationalisierungsprozesses verkehrt, oder es wird sogleich eine nächste Erklärungsmöglichkeit ins Spiel gebracht. Der Geistesmensch (und damit auch der Leser) gerät dadurch in immer neue Verstrickungen und Widersprüche hinein. Als Lebenslügner verfügt er über hohe intellektuelle Fähigkeiten, andernfalls wäre er gar nicht in der Lage, den täglichen Kampf gegen Fakten und Einsichten erfolgreich zu bestehen: Ihre Anordnung und ihr vorgeblicher Zusammenhang ist hier Konstruktion. Der Mechanismus ist dabei immer derselbe: Das für die Studie unbedingt als erforderlich Postulierte wird zur gleichen Zeit für das Scheitern des Projekts verantwortlich gemacht: die Enklave bzw. die erzeugte Beziehungslosigkeit; die destruktiv einwirkende Masse bzw. die Gesellschaft; das ebenfalls mit potentieller Zerstörungewalt ausgestattete unmittelbare soziale Umfeld (BET 40); Kindheit und Erziehung; sogar die „sogenannte wunderbare Natur“, die als bedrohlicher Gegenpol „den Kopf absolut [schwäche]“ (KA 216); und alle Lebenstätigkeiten, auch Bücherlesen und Gedankendenken. Überhaupt „alles sei“, so der Geistesmensch Konrad im Kalkwerk, ,gegen die Niederschrift“ (KA 48). Noch problematischer wirkt aber diejenige Ablenkung, die nicht von außen kommt, sondern vom Geistesmensch selbst hervorgerufen wird: „gleich was als Ablenkung sei ihm recht, alles sei ihm recht und nichts lächerlich und nichts minderwertig und unbedeutend und desavouierend genug, um sich abzulenken, um sich nicht mit der Niederschrift der Studie befassen zu müssen [...]“(KA 126). Auf diese Weise versucht der Geistesmensch, sich für einige Augenblicke von seiner Lebenslüge zu befreien. Denn das Denken an die Niederschrift der Studie, um die er sich jahrzehntelang bemühte, ist mittlerweile „das Entsetzlichste“ (KA 126) geworden.

Der Geistesmensch tut alles, um seinen Glauben an die höhere Bedeutung und Einzigartigkeit seines Lebens aufrechtzuerhalten, aber er tut nichts, um diesen Glauben zu rechtfertigen. Anstatt sich ernsthaft um die Beseitigung der äußeren Hindernisse zu bemühen, ist er darauf bedacht, es nie zu einer Entscheidung kommen zu lassen. Der hohe Wert seines Lebens besteht eigentlich nur der Möglichkeit 
nach, es ist eine Art Versprechen, dessen Einlösung an diverse Bedingungen geknüpft ist, die jedoch nie erfüllt werden. Der für die Niederschrift geeignete Zeitpunkt bleibt aus (KA 72) und zwingt, die Geistesarbeit ,durch bewußte oder unbewußte Verzögerungstaktik“ (KA 198) auf den ,richtigen Zeitpunkt“ (BET 206) zu verlegen bzw. „Jahrzehnte lang vergeblich auf den idealen Moment“ (KA 269) zu warten, schließlich würden die Sprache und „[d]ie Wörter ruinieren, was man denkt" (KA 147). Die Lebenshaltung und Existenzerfahrung des Geistesmenschen entspricht daher dem ständigen Aufschub: Er schiebt die Entscheidung darüber, ob er auf intellektuellem (künstlerisch-wissenschaftlichem) Gebiet tatsächlich etwas zu sagen hat oder ob er ewiger Dilettant bleiben wird, immer wieder hinaus. Er hat Angst zu entdecken, dass er nicht außerordentlich ist:

An Rücksichtslosigkeit auch oder gerade gegen sich selber habe es ihm [...] nicht gemangelt, aber das Wichtigste habe ihm gefehlt: Furchtlosigkeit vor Realisierung, vor Verwirklichung, Furchtlosigkeit einfach davor, seinen Kopf urplötzlich von einem Augenblick auf den andern auf das rücksichtsloseste um- und also die Studie auf das Papier zu kippen. (KA 210-211)

So kann er sich der Illusion hingeben, die Apotheose seines Schaffens liege noch vor ihm, es bedürfe nur einer geringfügigen Willensanstrengung, zu der er ohne weiteres imstande wäre, um seine wahre Größe unter Beweis zu stellen. Sein Bild von ihm selbst ist das eines Menschen, der noch nicht am Ziel angelangt ist, der ,unterwegs', auf dem Weg zu sich selbst ist. Als Lebenslügner wartet er voller Sehnsucht auf das große Ereignis seines Lebens und tut doch alles, um zu verhindern, dass es jemals eintritt. Denn nur auf diese Weise kann er sich den Glauben an sich selbst und an die hohe Bestimmung seines Lebens bewahren. Ein ernsthafter Versuch, seine imaginäre Lebensaufgabe zu bewältigen, könnte fehlschlagen, und dieses Risiko will der Geistesmensch nicht auf sich nehmen. Viel lieber hält er an der Vorstellung fest, die Bewährungsprobe stehe noch bevor, und der eigentliche Sinn seines Lebens würde erst zu einem späteren Zeitpunkt offenbar werden. Sollte er die Grenzen des ihm Erreichbaren jemals anerkennen, müsste er sich sein Scheitern eingestehen, denn dann wäre es mit der Illusion, kein Alltagsmensch zu sein, vorbei.

Bernhards Geistesmenschen brauchen die Lebenslüge, um nicht an der Mediokrität und Banalität ihres Lebens zugrunde zu gehen. Der Autor stellt Männer dar, deren Lebenstragödie darin besteht, dass ihre Lebenslüge der heutigen Realität nicht länger standhält: Sie leiden an der Lebensuntüchtigkeit ihrer eigenen Lebenslüge. Es geht also dabei nicht nur, wie Alexandra Pontzen behauptet, um ein Modell negativer Produktionsästhetik, um die „Dingmetapher für die künstlerisch-kreative Ausgangssituation des Beginnens“ und um „die Faszination und den Schrecken des leeren, weißen Blattes" ${ }^{\text {"17 }}$. Bernhards Geistesmenschen versuchen vielmehr, sich selbst über den Sinn und Wert des eigenen Lebens zu täuschen. Das tun sie gleich in doppelter Hinsicht: zum einen weil sie sich eine falsche, irrige Vorstellung über das Ziel des eigenen Lebens machen, das sie darin sehen, der eigenen Person eine herausragende Bedeutung auf intellektuellen Gebiet zu verleihen, und zum anderen, weil sie sich über ihren Erfolg beim Erreichen dieses Ziels etwas vormachen. In

17 Pontzen, Künstler ohne Werk, S. 313. 
gewisser Hinsicht stellen sie dem eigenen Leben zwei negative Vorzeichen voran: Sie verfolgen das falsche Ziel, und sie erreichen es nicht. Sie glauben aber daran, dass es das richtige Ziel ist und dass sie es erreichen werden - jedenfalls wollen sie daran glauben und tun so, als würden sie daran glauben. Ihr Selbstbetrug hält sie davon ab, den Sinn des Lebens auf einem anderen Gebiet zu suchen oder zumindest ein Leben in der Wahrheit zu führen.

Die Lebenslüge ist ihre Strategie, deren Zweck darin besteht, den Zwiespalt zwischen dem Wunsch nach Abgrenzung von den anderen und dem Streben nach Bestätigung durch die anderen zu überwinden. Das bedeutet, dass sie sich nicht zufällig in eine Lebenslüge verstrickt haben, sondern dass diese ihr Ausweg aus der Krise, aus der Einsicht in die Nichtigkeit und Sinnlosigkeit des Daseins - ihres Daseins - ist. Die Lebenslüge ist ihr Rettungsanker im Strom des Lebens. Bernhards Geistesmenschen sind somit dramatische Figuren, deren Tragik eher belustigend wirkt, denn auch ihr tragisches Schicksal ist Teil ihrer Lebenslüge. Ihre eigentliche Leistung besteht darin, den Selbstbetrug in den Rang einer metaphysischen Aufgabe des Lebens erhoben zu haben. Bernhards Geistesmenschen beweisen in und mit ihren Projekten, unabhängig von deren Gelingen, sich selbst ihre Außerordentlichkeit. Ihre Lebenslüge ist die heroisierende Mystifikation eines Versagens. Sie stehen einer feindlich gesinnten, verständnislosen „Umwelt“ (KO 219) gegenüber, die das Genie naturgemäß verkennt:

[...] es waren ihm von allen Seiten Vorwürfe gemacht worden, daß er überhaupt in einer Zeit gegen solche Ideen eine solche Idee habe [...], daß er in einer Zeit, welche ja überhaupt gegen solche Menschen und Köpfe und Charaktere und Geister wie Roithamer (und andere!) sei, [...] ein solcher widersprüchlicher Charakter und Geist und Mensch noch dazu, welcher sein ihm auf einmal zugefallenes Erbe dazu benutzte, einer, wie alle sagten, verrückten Idee zu gehorchen, die auf einmal in seinem verrückten Kopfe aufgekommen sei (KO 18).

In ihrem einsamen und unverstandenen Kampf leisten sie mit Hilfe des „Verstandes“ einen Alleingang, der sich als „Zuwiderhandlung“ (KA 130) definiert und zu deren Attributen eine eminente Größe gehört: „Es erfordere eine beinahe übermenschliche Anstrengung, immer gleichzeitig alles zu sehen [...]“" (KA 116). So findet jene Allianz von Männlichkeit, Unternehmungsgeist und Individuierung Ausdruck, die sie unwiderruflich von der Frau trennt.

Bernhard inszeniert die Konstruktion des traditionellen Geschlechterdiskurses als Kampf zwischen dem bedrohten und männlich besetzten ,Geist' und der als übermächtig wahrgenommenen weiblichen ,Geistesfeindlichkeit'. Denn Frauenfiguren fordern Eindeutigkeit, sei es die der Niederschrift oder die des Werkverzichts. Frau Konrad z.B. ,glaube ja nicht an das, was er ihr täglich zu beweisen versuche und was sich ganz einfach nicht beweisen lasse, daß er eine grundlegende, er scheute sich in letzter Zeit, aus Verzweiflung [...], gar nicht zu sagen epochenmachende Studie im Kopf habe.“ (KA 146) Eine Studie, die nur im Kopf existiert, verkörpert in den Augen der Frau Konrad das Widervernünftige und Irreale. Sie steht verständnislos vor dem Ausbleiben der Niederschrift:

[...] jeden Tag sage er seiner Frau, daß die Studie zwecklos sei, solange er sie nur im Kopf, nicht aber auf dem Papier habe und sie sage immer, warum er sie dann nicht 
endlich aufschriebe, jahrelang sagt sie das in dem immer gleichen Tonfall [...], weil sie noch immer nicht begriffen habe, daß man eine Studie durchaus jahrelang und, wie ich weiß, jahrzehntelang im Kopf haben kann, ohne sie zu Papier bringen zu können. Darin seien alle Frauen gleich, daß sie Merkwürdigkeiten wie diese nicht begreifen [...]. (KA 67)

Folglich bezeichnet sie den Geistesmenschen als „Narren“ und „Verrückten“ (K 167), seine Studie als „Hirngespinst“ (K 146). Das Verhältnis zwischen Frau und Geistesmenschen ist von Unverständnis und Ignoranz geprägt (KA 186). In Beton beschreibt Rudolf seine Schwester als „das geistfeindlichste Wesen, das sich denken läßt“ (BET 13) ${ }^{18}$, als einen „bösartige[n]“ und „hinterhältige[n]“ (BET 14) Menschen, „dessen Lebensaufgabe es zu sein scheint, zu stören, alles und jedes zu stören und damit zu zerstören und letzten Endes zu vernichten und immer wieder das zu vernichten, was [ihm] als das allerwichtigste erscheint auf der Welt: ein Geistesprodukt" (BET 14-15).

Der Natur des männlichen Wesens sei ,angeboren, was der Frau angelernt werden müsse in mühevoller, oft verzweifelter Lernmethode, nämlich der Verstand als chirurgisches Instrument gegenüber der sich sonst unweigerlich auflösenden, ja sonst rettungslos zerbröckelnden Geschichts- und Naturmaterie“ (KA 129). Der Verstand als männliche Kraft hängt dafür mit den wissenschaftlichen Lektüren zusammen, die „in Beziehung zu allem Möglichen“ (KA 129) offen erscheinen. Bernhard folgt hier einer Tradition, so hat Mittermayer gezeigt, die bereits zu Beginn des Jahrhunderts in der ,prinzipiellen Untersuchung“ Geschlecht und Charakter von Otto Weininger Niederschlag gefunden hat - und nach der das, Weib' zum Gegenpol von den männlich belegten Instanzen ,Geist' und ,Verstand' stilisiert wird. ${ }^{19}$ Weininger hatte dort nämlich behauptet, dass „Genialität an die Männlichkeit geknüpft ist, daß sie eine ideale, potenzierte Männlichkeit vorstellt ${ }^{\text {‘20 }}{ }^{2}$. Genau diesem klischierten Denkmuster fügt sich der Geistesmensch Roithamer in Korrektur, indem er verallgemeinernd behauptet, es sei ganz natürlich, „,daß das weibliche Geschlecht, [...], gegen den Geist und nur aus dem Gefühl und zwar gegen den Geist in allen seinen Möglichkeiten wie für das Gefühl aus dem Gefühl in allen Möglichkeiten“ sei; und außerdem wisse er „aus Erfahrung, daß der weibliche Mensch [...], daß das weibliche Geschlecht über eine erste Willigkeit zum Geistigen nicht“" (KO 317) hinauskomme. Auf der einen Seite steht der ,Instinkt-' bzw. ,Gefühlsmensch' mit seiner „Ungeistigkeit“ (KO 298) und Dummheit (KO 250) bzw. seiner krankhaften Abneigung gegen „Papier“, „Zeitschriften“ und „Bücher" als „Geistesgrundlage“ (KO 298-299) und auf der anderen der Geistesmensch, dessen Selbstverständnis mit solchen Begriffen wie Kopf, Denken, Vorstellung, Idee, Phantasie, aber auch Kunst und natürlich Geist assoziiert wird ${ }^{21}$ - „Geistigkeit“ gilt hier als „männliche Kopfverfassung" (KO 298-299).

${ }^{18}$ Zur Geistfeindlichkeit der Frau vgl. auch BET 12, 13f., $18 \mathrm{f}$.

19 Vgl. Manfred MittermaYer, Ich Werden. Versuch einer Thomas-Bernhard-Lektüre, Stuttgart, Heinz, 1988, S. 55f.

${ }^{20}$ Otto WeInINGER, Geschlecht und Charakter. Eine prinzipielle Untersuchung [1903], München, Matthes \& Seitz, 1997, S. 144.

${ }^{21}$ Vgl. KO 9-12, 18, 27-28, 30-31, 58, 60, 80, 158, 227-228, 245, 301, 310, 318. 
Während der Geistesmensch Konrad sich sagt, „die Gesellschaft ist nichts, die Studie ist alles“, beharrt seine Frau auf der Formel, „die Studie ist nichts, die Gesellschaft ist alles“ (KA 137). In Beton wird der Protagonist schließlich von den Ausführungen einer Frau, d.h. seiner Schwester angestoßen und muss die Lebenslüge als den eigentlichen Antrieb seiner Geistesambitionen zugeben: „sie hat vielleicht recht, indem sie sagt, meine Arbeit über Mendelssohn Bartholdy ist nur eine Finte, um meinen absurden Lebenswandel $\mathrm{zu}$ rechtfertigen, der, außer, daß er etwas schreibt und vollendet, keine andere Rechtfertigung hat" (BET 70). Der Geistesmensch muss gestehen, dass tatsächlich alle seine schriftstellerischen, künstlerisch-wissenschaftlichen Versuche dem gleichen Zweck gedient haben:

\begin{abstract}
Ich stürzte mich auf Schönberg, um mich zu rechtfertigen, auf Reger, auf Joachim, ja sogar auf Bach, nur um mich zu rechtfertigen, wie ich mich jetzt auf Mendelssohn stürze zu demselben Zweck. Im Grunde habe ich überhaupt kein Anrecht auf meine Art von Lebenswandel, der tatsächlich so einmalig wie kostspielig und genauso fürchterlich ist. (BET 70)
\end{abstract}

Da Rudolf weder in seinen Handlungen noch in seiner vereinsamten Lebensführung einen rechten Sinn erkennen kann, ihm aber gerade wegen seines isolierten Zustands nichts anderes als die Studie geblieben ist, sieht er sich dazu genötigt, seine Lebenslüge aufrecht zu halten und seinen unbedingten ,Kunstwillen’ zu simulieren: „Es war das beste, die Frage nach dem Sinn oder Unsinn einer solchen Arbeit weiter zu stellen, aufzugeben und ich gab sie auf und tat so, als sei ich entschlossen, die Arbeit tatsächlich so bald als möglich anzugehn." (BET 174) Im Verlauf seiner selbst-kritischen Überlegungen wird sich der Geistesmensch Rudolf nicht nur über die ,theatralisch[e]“ (BET 114), sondern auch über die komische Seite seiner Situation bewusst; er sei in seiner „eigenen Komödie“ (BET 118) gefangen, schreibt er. So schlagen die Allmachtsphantasien des Geistesmenschen am Ende in die Ohnmacht des nach Herrschaft Ringenden um: „Man beherrsche nichts, mißbrauche alles“ (K 149).

Bernhard, der als schreibender Patriarch bzw. Verfasser von frauenfeindlichen Texten gilt, betreibt in diesem Sinne weniger eine bejahende Reproduktion des Geschlechterdiskurses als vielmehr dessen subtil subversive Zersetzung. ${ }^{22}$ Besonders auffällig geschieht dies in Korrektur. Hier hat sich der Geistesmensch Roithamer dazu entschlossen, ein kegelförmiges Kunstwerk zu bauen, von dessen Verwirklichung er sich das Glück (KO 223) und die endgültige und unwiderrufliche Überwindung seines Herkunftskomplexes (KO 225) verspricht. Nachdem er seine Schwester, die sich ,vor diesem Augenblick [...] gefürchtet“ [...] habe, in den Kegel hineingeführt hatte, sei sie nicht voller Glück gewesen, sondern bald darauf gestorben. Ria Endres meint, dass das Bauwerk als ein „mathematischer Phallus“ zu deuten sei und der Kegel eine „Opferstelle männlicher Genialität“23 symbolisiere. In diesem Sinne wird die Gleichsetzung des Männlichen mit dem ,Gesunden', ,Potenten'

${ }^{22} \mathrm{Vgl}$. Verena Ronges, Ist es ein Mann? Ist es eine Frau? Die (De)Konstruktion von Geschlechterbildern im Werk Thomas Bernhards, Köln/ Weimar/ Wien, Böhlau, 2009.

${ }_{23}$ Ria ENDRES, Am Ende angekommen: dargestellt am wahnhaften Dunkel der Männerporträts des Thomas Bernhard, Wien/ Linz/ Weitra/ München, Bibliothek der Provinz, 1994, S. 65f. 
und somit die tradierte - implizite - Männlichkeit des ehemals im gottgleichen Schöpfertum verankerten Genies dekonstruiert. Die große Schöpfung bleibt aus, die Geistesmänner sind eigentlich krank, steril, unzeitgemäß, im besten Fall lächerlich, im schlimmsten Fall tödlich. Aufgebaut auf einer Lebenslüge erhellt sich ihre geistige Existenz als ausweglos; Rückzug und wissenschaftlich-philosophisch-künstlerische Arbeit bedeuten in letzter Konsequenz Selbstvernichtung, münden in tödliche Erschöpfung, Wahnsinn, Selbstmord, obwohl sie doch Selbstschöpfung bezweckten. Das männliche Subjekt erscheint dabei bestimmt über die Relationen von Substanz und Mangel, Ermächtigung und Unterwerfung. Es oszilliert zwischen den Extremen eines substantiellen Zentrums und absoluter Leere, kurz: Es wird als Angelegenheit von Leben und Tod bzw. alles oder nichts verhandelt. Denn in dem Maße, wie sich das (männliche) Bewusstseins-Subjekt zum Herrscher der Welt erklärt, findet es sich, so die wirkungsreiche Argumentation von Theodor W. Adorno und Max Horkheimer, in eine Dialektik aus Selbstermächtigung und Selbstunterwerfung verstrickt, entwickelt sich zum Akteur und zum Opfer seiner eigenen Unterdrückung. ${ }^{24}$ Michel Foucault entfaltet aus genealogischer Sicht ein ganz ähnliches Konzept des Subjekts, das sich als ebenfalls ,unterworfenes” hervorbringt. ${ }^{25}$ Weltaneignung und Weltabwendung, Emanzipation und Verlust erscheinen also letztlich als die beiden Seiten, die für die moderne Subjektivität konstitutiv sind. Eine stabile männliche Identität ist eine Illusion, sowohl die negative Weiblichkeitsimago als auch die Autonomie des Geistesmenschen sind imaginäre Konstrukte. Bernhards Texte legen in diesem Sinne Mechanismen und Bedingungen offen, durch die die traditionelle Geschlechterkonstruktion mit dem Anschein von Naturhaftigkeit überzogen wird. Sie verlieren somit ihre Wirkmächtigkeit, um eine zweite Wahrheit erkennen zu lassen - die Wahrheit über die Künstlichkeit des Geschlechterdiskurses.

${ }^{24}$ Max Horkheimer / Theodor W. Adorno, Dialektik der Aufklärung [1969], Frankfurt a. M, Fischer, 1988, S. 40 und S. 62f.

${ }^{25}$ Michel Foucault, Überwachen und Strafen. Die Geburt des Gefängnisses, Frankfurt a. M, Suhrkamp, 1977. 
